\title{
Memory editing from science fiction to clinical practice
}

Elizabeth A. Phelps ${ }^{1,2} *$ \& Stefan G. Hofmann ${ }^{3}$

\begin{abstract}
Science fiction notions of altering problematic memories are starting to become reality as techniques emerge through which unique memories can be edited. Here we review memory-editing research with a focus on improving the treatment of psychopathology. Studies highlight two windows of memory vulnerability: initial storage, or consolidation; and restorage after retrieval, or reconsolidation. Techniques have been identified that can modify memories at each stage, but translating these methods from animal models to humans has been challenging and implementation into clinical therapies has produced inconsistent benefits. The science of memory editing is more complicated and nuanced than fiction, but its rapid development holds promise for future applications.
\end{abstract}

I f you could erase the memory of the worst day of your life, would you? How about your memory of a person who has caused you pain? The notion that this is possible was the premise of the critically acclaimed film Eternal Sunshine of the Spotless Mind,

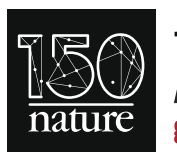
150 YEARS OF NATURE Anniversary collection go.nature.com/nature150 as optogenetics show considerable promise for the editing of emotional memories in animal models ${ }^{5,6}$, these approaches are too invasive for use in the clinic. So far, a range of techniques have been identified that can be used to edit unique human memories, but in which two lovers choose to erase their memories of a relationship that has gone bad, only to find that they are drawn to each other once again. In other science fiction films, memories are erased to hide government secrets (Men in Black) or implanted to provide virtual 'vacations' (Total Recall). The idea of selectively editing memories has also emerged in children's films (The Incredibles 2, Frozen). As these films illustrate, even when the intention of memory editing is to reduce pain or protect someone, there can be unexpected consequences. The memory-manipulating techniques in these films rely on science fiction-based notions about how we can edit memories, with the implicit assumption that unique memories have identifiable signatures in the brain that can be targeted for implantation or deletion.

Despite the prevalence of memory editing in science fiction, it has mostly been just that-fiction. Although the identification of synaptic changes that underlie the representation of simple associative memories in invertebrates demonstrated that it is possible to alter the neural instantiation and behavioural expression of unique memories ${ }^{1}$, applying similar techniques to humans has proved elusive. This is in part because the neural representations of memories are far more complex in vertebrates, and also because methods that have been used to alter synaptic plasticity in animal models are not safe for use in humans. However, neuroscientists and psychologists have begun to investigate techniques that may overcome these challenges. These new approaches are being used to address the prospect of purposefully editing human memories, with goals such as reducing the emotional consequences that stem from memories of traumatic events ${ }^{2}$, diminishing cravings that are induced by drug cues in addicts $^{3}$ or enhancing education ${ }^{4}$.

This Review describes recent advances in memory editing and how they might translate to current clinical practice. For this reason, we focus on memories for events with an emotional meaning, as these are more likely to be clinically relevant. Because we are interested in the editing of unique memories, we do not discuss techniques that enhance or impair memory broadly. In addition, we primarily examine approaches that have been or can be used in humans. Although techniques such attempts to translate these to clinical therapies have produced inconsistent benefits. Translation from animal models to human memories has been challenging because interventions used in animals are often unsafe for people, and human memories are more complex. The science of memory editing is nuanced and complicated, and relatively little is known at present about how these techniques might most effectively be applied to clinical treatments. However, the rapid speed at which this science is advancing suggests the potential for promising applications of memory-editing techniques in the future $\mathrm{e}^{7,8}$.

\section{The science of memory editing}

Challenges and windows of vulnerability

One challenge of applying memory-editing research to the clinic is that a memory for a single event can be expressed in several ways, each of which is linked to a distinct neural representation. The depiction of memory editing in science fiction mainly highlights efforts to alter the conscious recollection of life events, which is known as episodic memory. However, a traumatic event-a car accident, for exampleproduces multiple forms of memory expression. The victim will probably consciously recollect details such as where and how the accident happened. In addition, exposure to an accident cue (such as seeing the street corner where it occurred) may evoke momentary freezing and physiological arousal, or learned defensive responses. The person may also habitually avoid that corner. Finally, reminders of the accident may evoke negative subjective feelings. Although these different forms of memory for the same event (that is, episodic details, defensive responses, habitual actions and subjective feelings) may interact, each involves a distinct neural system for storage and expression. For this reason, targeting one type of memory representation for editing may or may not alter other forms of memory for the same event. This specificity can have advantages and disadvantages. For example, it might be advantageous to retain accurate conscious memory for the details of an event, but edit the associated negative feelings or defensive responses. Conversely, it could be problematic to retain defensive responses, 
habitual actions and negative feelings that are linked to a traumatic event that can only be consciously recalled to a limited extent. Researchers generally attempt to edit only one form of memory, so it is not always clear how other forms of memory for the same event may be affected. Most current research focuses on two types of representation: episodic memory (mediated by the hippocampus); and defensive responses (mediated by the amygdala). By contrast, most clinical interventions focus on reducing maladaptive habits and negative feelings that are associated with psychopathologies (see Box 1). In this Review, we will highlight which type of memory expression is targeted for each of the editing techniques discussed.

By definition, a technique that is used to edit a memory has to do so after the memory is initially formed, or encoded. Because of this, memoryediting techniques are generally thought to work either by modifying the initial storage of a memory (consolidation), or by modifying its re-storage after retrieval (reconsolidation) (Fig. 1). Initial consolidation can be prolonged, particularly for hippocampal-dependent episodic memories ${ }^{9}$, so it can be hard to tell whether a technique is targeting consolidation or reconsolidation-especially in humans, as markers of synaptic plasticity that signal memory storage cannot be assessed. Similarly, it can be difficult to differentiate the editing of a memory representation from the formation of a new memory that competes for expression with the older one.

Another challenge in targeting unique memories for editing is that, with rare exception, the neural signatures of even simple memories are complex and can be distributed throughout the brain, making it impractical to mechanically identify and target them for editing. To address this problem, researchers have relied on the finding that neural ensembles that represent unique memories are active at certain times and thus may be vulnerable to editing during these specific periods. One such time is during the initial consolidation of memories, which is a gradual process of transformation and stabilization that entails recurrent neuronal reactivations of the memory both while a person is awake and asleep. This process-and memory editing that is thought to target consolidation-has been studied most often in the context of hippocampal-mediated episodic memories ${ }^{9}$. A few editing techniques that are proposed to alter the consolidation of unique memories have shown evidence of success in humans.

\section{Editing memory consolidation}

In animal models, administering amnestic agents (typically inhibitors of protein synthesis) after learning can prevent the synaptic changes that are required for the consolidation of memories and induce the forgetting of recently encoded memories. These agents have no clinical potential, as they non-specifically impair memory for all recently encoded events and are generally not safe for use in humans. More promisingly, endogenous neurohormonal changes also alter the consolidation of memories, particularly for emotional events. Research in this area ${ }^{10}$ shows that stress hormones evoked by emotional events enhance consolidation and thereby strengthen memories for these events. Drugs that are safe for human use can mimic (for example, adrenaline and glucocorticoids) or block (for example, propranolol) this effect of stress hormones. These drugs work through $\beta$-adrenergic receptors in the amygdala, which then modulates hippocampal consolidation. In humans, events that are emotionally arousing are associated with enhanced memory consolidation, relative to neutral events ${ }^{11}$, and inducing arousal after encoding can also improve memory for events that have an emotional meaning ${ }^{12}$. In addition, systemic administration of propranolol, a $\beta$-adrenergic antagonist, reduces the emotional strengthening of episodic memory ${ }^{13}$.

Manipulating stress reactions during consolidation has the potential to affect clinical outcomes in positive ways, but it may also have adverse effects. One potential negative clinical consequence is that immediate processing of the trauma memory might strengthen it. Studies examining the effect of psychological debriefing of acutely traumatized individuals found that talking about the trauma experience soon afterwards can result in worse clinical outcomes weeks to years later,

\section{Box I The feelings of memories}

Memories for traumatic events are clinically important in part because their recollection reinstates some of the negative feelings and reactions that are associated with the original event. Whether or not a memory is accurate in its episodic details is less important than the emotional effect that accompanies its recollection. However, few laboratory studies of memory editing have examined the feelings of memories (except for feelings of craving). Although researchers have used the recollection of memories to evoke subjective feelings in experimental manipulations that change moods ${ }^{85,86}$, reduce stress ${ }^{87}$ or bias decisions ${ }^{88}$, investigations of the mnemonic processes that underlie these subjective feelings are less common. So far, three lines of research have explored topics that are relevant for understanding the feelings of memories.

Studies of evaluative conditioning examine how subjective feelings or attitudes of liking and pleasantness are acquired through association. In a typical study, neutral stimuli (such as an office scene) are paired with emotional stimuli (such as an accident scene or a picture of a puppy). After pairing, participants are asked to rate their attitude towards the neutral stimuli. Relative to unpaired neutral stimuli, those paired with positive or negative stimuli are rated as more liked/pleasant or more disliked/unpleasant, respectively ${ }^{89}$. Notably, although learning through association with an emotional event also underlies Pavlovian aversive and appetitive conditioning, learned defensive and appetitive responses can be diminished through extinction techniques, but these are less effective for changing subjective evaluations ${ }^{90}$. By contrast, other manipulations may be more effective $e^{91}$ or equally effective ${ }^{92}$, suggesting that different factors may mediate the alteration of these different forms of expression of emotional, associative memories.

Studies of emotion regulation typically focus on cognitive strategies to alter feelings about current events, but a few studies have explored how techniques of regulation can change the feelings of memories ${ }^{93}$. For example, patients with major depressive disorder show an increase in negative feelings (relative to healthy controls) when recalling negative autobiographical events, but these patients are equally effective as control participants at using a strategy of cognitive regulation to diminish the negative feelings. Furthermore, patients and healthy participants engage overlapping brain circuits that are involved with the regulation of emotions ${ }^{94}$.

This research suggests that it is possible to use standard techniques of emotion regulation to specifically target the feelings of memory.

One outstanding question is whether the feelings of memories accurately reflect the emotional experience of the original event. To our knowledge, there is no research on this topic, but a study of memory for the terrorist attack of 11 September 2001 examined the consistency of memory details over time, including how the participant felt at the time of the attack ${ }^{95}$. In line with previous studies of flashbulb memories, about $40 \%$ of the episodic details (for example, responses to the question 'How did you hear about the attack?') changed over time, despite high confidence that the memory was accurate. Of note, consistency in memory for emotional reactions was worse than for episodic details, with $60 \%$ of responses changing over time. Although the reasons for poorer memory for feelings are unclear, the shifting nature of these memories suggests they might be easier to modify than other aspects of memory expression.

in comparison to traumatized individuals who did not undergo psychological debriefing ${ }^{14,15}$. Although it is unclear how psychological debriefing exerts negative effects, it is hypothesized that debriefing induces a secondary traumatization, which may enhance the consolidation of the traumatic memory. Conversely, as propranolol is well-tolerated and 


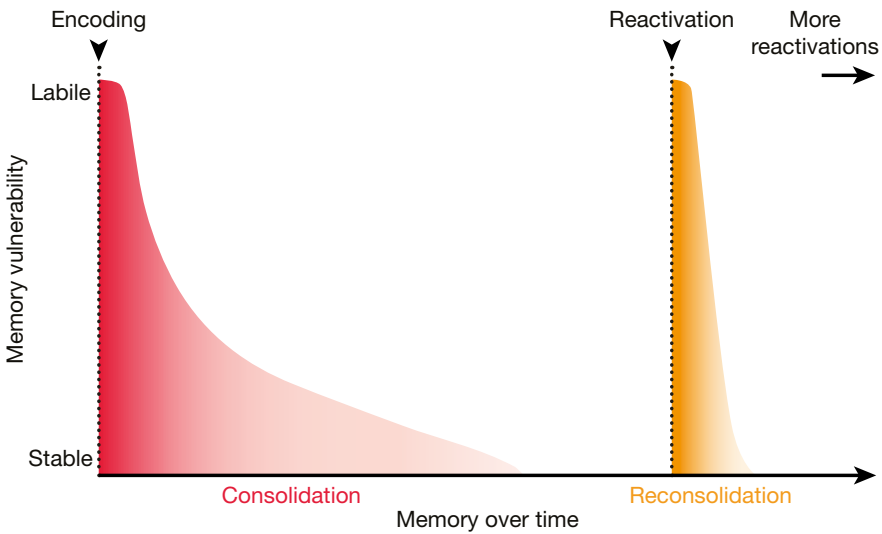

Fig. 1 | Windows of memory vulnerability. There are two time windows when memory may be labile and vulnerable to editing: initial consolidation (red); and reconsolidation following reactivation (orange). Memory consolidation can be a relatively long process, but most memoryediting techniques target memories minutes to a day after their initial encoding. Techniques targeting memory reconsolidation are generally effective for minutes up to a few hours after memory reactivation.

readily available, researchers investigated whether acute administration of propranolol after a traumatic event could diminish the consolidation of the memory and the likelihood of developing post-traumatic stress disorder (PTSD). Although this treatment reduced some indications of physiological arousal when study participants later consciously recollected the traumatic event, there was no effect on the likelihood of developing symptoms of PTSD ${ }^{16}$.

Slightly more success was obtained in studies that examined whether glucocorticoids could be used to augment exposure therapy as a treatment for phobia ${ }^{17}$ and PTSD ${ }^{18}$. Exposure therapy is based on the principles of extinction learning, in which repeated exposure to a threatening stimulus (or memory) with no aversive consequences results in the subject learning that this stimulus is now safe. Notably, extinction learning does not alter the strength of the original threat or trauma memory, but rather results in the formation of a new 'safe' memory that competes for expression with the threat memory ${ }^{19}$. Successful expression of extinction learning requires prefrontal inhibition of the amygdala to reduce the expression of the threat memory ${ }^{20}$. Because threat learning is generally strong and extinction learning is weaker, the threat memory can return even when extinction is initially successful, which may partially explain the relapse of clinical symptoms following exposure therapy ${ }^{21}$. In an effort to strengthen the consolidation of a safe memory, glucocorticoids were administered before exposure therapy. Clinical symptoms were reduced, relative to a placebo control group, up to six weeks later. However, glucocorticoids both impair retrieval of emotional memories and enhance consolidation of new learning, so it is unclear to what extent each mechanism accounts for the results. Administration of glucocorticoids after exposure therapy for PTSD, to target consolidation, produced a short-term benefit ${ }^{22}$.

Another way to enhance the initial consolidation of unique episodic memories is to influence their reactivation by external cues. Consolidation is thought to entail recurrent reactivations of the neuronal ensemble that represents the memory. These reactivations generally occur outside of consciousness and are proposed to be important in the stabilization and integration of memories ${ }^{9}$. Targeted memory reactivation (TMR) alters the reactivation of memories to influence the strength of consolidation. Evidence in humans shows that presenting cues linked to a previously encoded event during periods of sleep or awake rest produces blood oxygenation level-dependent patterns of activity in the hippocampus that are consistent with the reactivation of neuronal memory, and also strengthens later episodic memory for these events ${ }^{23-25}$. Most studies of TMR assess memories for neutral events, but the effect of TMR during sleep may be stronger for emotional, arousing stimuli ${ }^{26}$.
A few studies have examined how TMR affects amygdala-mediated learned defensive responses in humans. These studies used a Pavlovian aversive conditioning procedure in which odours are paired with shock and as a result of this pairing elicit a defensive response. The odours are then presented again during sleep. In contrast to episodic memories-which are strengthened by TMR-presentation of these odours during sleep diminishes learned defensive responses ${ }^{27,28}$, possibly by promoting extinction learning. Given this, it is unclear whether TMR during sleep edits the original threat memory or produces a competing, safe memory.

Although TMR is typically used to strengthen episodic memory, a recent study demonstrated that it is also possible to use TMR to impair episodic memory by cueing study participants during sleep with a tone linked to an intention to forget ${ }^{29}$. This study combined TMR with another class of memory-editing techniques often called motivated or directed forgetting, which we will broadly term 'memory control'. Such techniques use the intention to forget some events, or suppress their retrieval, to diminish later episodic memory. For example, in retrieval suppression, participants learn cue-target pairs and are then given the cues again with instructions to either retrieve or suppress retrieval of the target memory. In a subsequent test, episodic memory is worse for suppressed targets than for baseline targets. The amount of forgetting increases with the number of times a memory was suppressed, indicating that unwanted memories are cumulatively inhibited or forgotten over repeated suppressions ${ }^{30}$. Individual variability on this task is related to affective traits; poor performance is linked to PTSD, higher trait anxiety, more rumination and greater memory intrusions after viewing a disturbing film clip ${ }^{30}$. In addition, healthy individuals with a history of trauma are more successful in retrieval-suppression tasks than those with little or no trauma exposure-perhaps because the individuals who were exposed to trauma had developed skills in memory suppression ${ }^{31}$.

Neuroimaging studies of memory control show that these techniques engage prefrontal brain circuits that are typical of effortful control, which might underlie inhibition or suppression ${ }^{30}$. These studies also show reduced blood oxygenation level-dependent activity in the hippocampus and changes in a hippocampal marker of the neurotransmitter GABA ( $\gamma$-aminobutyric acid), which indicates GABAergic inhibition of hippocampal retrieval processes ${ }^{32}$. These findings are consistent with inhibition of hippocampal consolidation, which may promote forgetting and editing of memory strength. Current approaches in humans cannot fully discern whether techniques of memory control promote forgetting or the repression of conscious memory expression without editing. Supporting the view that the memory is edited, however, non-conscious access to it is also impaired after memory control ${ }^{33}$.

A final technique to modulate episodic memory consolidation is behavioural tagging, which is based on the premise of synaptic tagand-capture models in which memories that are initially weak are strengthened by the engagement of common neural pathways minutes to hours later ${ }^{34}$. In humans, if after a stimulus is encoded, conceptually related stimuli are subsequently made relevant by pairing with shock ${ }^{35}$ or monetary reward ${ }^{36}$, later memory for the initially encoded stimulus is enhanced relative to conceptually unrelated stimuli that were learned at the same time. So far, evidence for behavioural tagging is sparse in humans, and research on its neurobiological underpinnings is limited to animal models ${ }^{34,37}$

The memory-editing techniques that alter consolidation show that there are many potential ways to enhance the strength of a unique memory and a few ways to weaken it. One limitation of these techniques for clinical use is that consolidation is time-restricted to soon after initial learning 9 . Precisely how long episodic memory consolidation continues in humans is unclear, but the time window for successful application of some approaches (neurohormonal arousal, behavioural tagging) is limited to minutes to hours after encoding. Other approaches (TMR, memory control) may have a slightly longer time window, but have not been tested beyond a few days. However, people do not usually seek help until long after a distressing event 
occurred. At this time, memories for the clinically relevant event are presumed to be fully consolidated, so editing the memory by targeting consolidation would have little success.

For this reason, investigators are increasingly looking at techniques to edit memory reconsolidation. In reconsolidation, a previously consolidated memory becomes labile and prone to disruption or modification following reactivation or retrieval. Like consolidation, reconsolidation requires synaptic plasticity for the re-storage of the memory. Despite some differences in the timing and neuronal mechanisms that underlie initial consolidation and reconsolidation, as well as cases in which they cannot be clearly distinguished ${ }^{38}$, the idea that memories can-long after initial encoding-be edited through reactivation holds promise for clinical interventions that may not be available until months or even years after an important or traumatic event.

\section{Editing memory reconsolidation}

Studies of reconsolidation date back half a century ${ }^{39}$, but interest in this topic was reinvigorated when an experiment showed that learned defensive responses in rodents could be eliminated by targeting reconsolidation ${ }^{40}$. Using a Pavlovian aversive conditioning procedure, the researchers first created a threat memory in which a tone predicted a shock; subsequently the tone alone elicited a learned defensive response. After the threat memory had been consolidated, they reactivated it by playing the tone to trigger reconsolidation. A subsequent injection of an inhibitor of protein synthesis into the amygdala-the site of storage for these simple threat memories-blocked the synaptic plasticity necessary for the reconsolidation of this memory. A day later, when the tone was played again, the rats who had received the injection failed to show the learned defensive response, consistent with memory impairment or erasure.

The idea that one could essentially erase previously consolidated threat memories - at least as expressed through defensive responseswas of great interest to researchers and clinicians. Yet they struggled to find ways to translate these findings, because the amnestic agents that are typically used in animal studies are not safe for use in humans. After a follow-up study found that propranolol may also be effective at blocking the reconsolidation of threat memories in rodents ${ }^{41}$, several laboratories attempted to use propranolol to block the reconsolidation of learned defensive responses in humans, with mixed success ${ }^{42}$. When propranolol did eliminate expression of the threat memory, only some types of learned defensive responses were affected, and others did not change ${ }^{43}$. Researchers then attempted to diminish PTSD by influencing memory reconsolidation, but propranolol administered after the reactivation of a traumatic memory had no effect on PTSD symptoms ${ }^{2}$. Mifepristone, a glucocorticoid antagonist, was also ineffective ${ }^{2}$. Giving propranolol after the presentation of a feared stimulus reduced symptoms of phobia ${ }^{44}$, but it is not clear whether this was because a unique threat memory was targeted or a generalized fear reaction was affected $^{45}$. When administered before memory reactivation, propranolol diminished PTSD symptoms more effectively, but these results could also be caused by altering memory retrieval or facilitating new learning ${ }^{46}$.

Inspired by work in animals on editing the reconsolidation of threat memories, researchers investigated whether similar approaches could be applied to appetitive, drug-associated memories. Appetitive learning procedures in rodents assess the learned association between a cue and a drug by examining measures such as preference for the location where the drug was administered and self-administration of the drug in the presence of the cue. Administering inhibitors of protein synthesis during reconsolidation impairs a range of appetitive behaviours ${ }^{47,48}$, consistent with the blocking of reconsolidation of the drug-cue memory. The efficacy of propranolol was also assessed in animal models, but it was found to reduce some measures of the appetitive memory and not others ${ }^{48,49}$. A few researchers have given propranolol to addicts following reactivation of the drug-cue memory and found that subjects reported reduced cravings initially; however, the effect was short-lived, and drug use did not decrease $e^{8,42,50}$.
Although studies of aversive learning provide a framework for translating reconsolidation research from rodents to humans, these studies find that even when human defensive responses are diminished, episodic memory remains intact ${ }^{51}$. In rodents, blocking reconsolidation with amnestic agents disrupts hippocampal-mediated memories ${ }^{52}$, but these paradigms are difficult to translate to humans. The few studies that have explored the possible influence of pharmacological interventions on hippocampal-mediated episodic memory in humans examined whether endogenous stress hormones can modulate reconsolidation in a similar manner to consolidation ${ }^{10}$. Post-reactivation stress enhanced later episodic memory ${ }^{53}$, suggesting that endogenous stress hormones may also modulate reconsolidation. In addition, propranolol, administered with memory reactivation, subsequently reduced the typical enhancement of memory for emotional stimuli. However, because propranolol was given before memory retrieval, this reduction might be due to retrieval impairment or new learning, rather than modulation of reconsolidation ${ }^{42}$. The only study, to our knowledge, that has demonstrated a physiological blockade of episodic memory reconsolidation in humans took advantage of the amnestic properties of electroconvulsive shock therapy ${ }^{54}$, which is known to also disrupt consolidation ${ }^{55}$.

Research in animal models has shown that blocking reconsolidation pharmacologically results in memory impairments that resemble the erasing of memories, which has led to excitement about the potential applications of these findings to humans. However, efforts to translate the results of animal experiments to healthy humans and the treatment of clinical disorders have been mixed. This difficulty in translation reflects several factors ${ }^{51}$, most notably that animal studies typically infuse inhibitors of protein synthesis into discrete neural regions. Systemic administration of safe dosages of alternative drugs in humans has not produced the same effects. The challenge of translating these pharmacological studies in animal models to humans has prompted investigators to consider alternative ways to influence memory reconsolidation.

Reconsolidation is proposed to have two adaptive functions: strengthening and updating ${ }^{52}$. The additional (re)consolidation that a reactivated memory undergoes is thought to strengthen the memory. In addition, if a memory is reactivated in a context in which there is new information available that is relevant to the meaning or purpose of that memory, the reconsolidation process incorporates that new information into the old memory. The notion that memories are dynamic and can change over time has been documented by psychologists for decades $^{56}$. Reconsolidation provides a mechanism that may facilitate the dynamic nature of memory by allowing it to be updated with new information that is available at the time of retrieval.

Taking advantage of this updating function, one study ${ }^{57}$ examined whether Pavlovian threat memories in rodents could be permanently altered by means of a behavioural intervention to insert new, safe information in place of the threat memory during reconsolidation. To do so, the researchers reactivated a tone-shock threat memory after consolidation by playing the tone. During the time window of reconsolidation, the rodents received extinction training in which the tone was presented alone. As mentioned above, extinction training is typically thought to produce a new, safe memory that competes for expression with the older threat memory-as shown by the later return of learned defensive responses and prefrontal inhibition of the amygdala to block the expression of the threat memory ${ }^{20}$. When the researchers first reactivated the threat memory to induce reconsolidation, and then gave extinction training, the defensive responses of the rodents did not return, suggesting that the threat memory had been modified. Furthermore, memory reactivation before the start of extinction training triggered synaptic plasticity in the amygdala, which is consistent with a reconsolidation mechanism. Notably, not every retrieval of a threat memory resulted in indications of synaptic plasticity and memory lability; instead, this depended on the timing between reactivation and the first extinction trial ${ }^{57}$. Because memory lability is critical for the success of interventions that target reconsolidation, subsequent research has attempted to determine when memory reactivation results 
in lability and reconsolidation, and when it does not. It has been suggested that memory reactivations that produce prediction errors ${ }^{58}$ (in which a prior expectation is violated), and the cause to which the memory reactivation is attributed ${ }^{59}$, have a role in determining whether or not the memory is labile and susceptible to modification by influencing reconsolidation.

The finding that reconsolidation of threat memories could be altered without drugs was quickly translated to humans. Extinction training during reconsolidation prevented the return of defensive responses for at least a year-consistent with a permanent alteration of the threat memory ${ }^{60}$. Neuroimaging studies of the retrieval-extinction paradigm showed less involvement of the prefrontal cortex relative to typical extinction ${ }^{61}$, in accordance with the hypothesis that the amygdaladependent threat memory was edited and prefrontal inhibition was therefore unnecessary. On the basis of the initial behavioural studies in humans, several research teams attempted to modify standard exposure therapy for phobia by reactivating the exposure cue before the treatment session. Results were mixed ${ }^{62}$, but one study showed improved outcomes a month later ${ }^{63}$. For PTSD and intrusive trauma memories, reactivation of the target memory before interfering memory manipulations or distracting games shows some efficacy ${ }^{64-66}$, but it is unclear whether reconsolidation is the mechanism ${ }^{8}$.

The success of the retrieval-extinction paradigm in editing threat memories prompted investigations into whether it could be applied to appetitive, drug-associated memories. Like threat memories, learned physiological and behavioural responses to drug cues extinguish if the cue is repeatedly presented without drug reinforcement. However, these responses are prone to relapse, suggesting that the memory of the drugcue association is inhibited but not edited by standard extinction training. By contrast, when extinction training was preceded by reactivation of the drug-cue memory, rodents showed little evidence of relapse for many drug-seeking behaviours ${ }^{3,67,68}$; relapse of drug self-administration was also diminished in comparison with standard extinction, but not eliminated ${ }^{3}$. In human heroin and nicotine addicts, self-reported craving was reduced for up to 180 days following the retrieval-extinction procedure $^{3,69}$, but although the nicotine addicts smoked fewer cigarettes one month later, their likelihood of relapse did not change ${ }^{69}$.

Although these simple appetitive and aversive learning studies suggest that the original memory has essentially been replaced, traces of it can be observed, which is consistent with updating and integration of old and new memories. For example, in a study of Pavlovian aversive learning using the retrieval-extinction procedure, whereas freezing was persistently reduced, rodents continued to suppress reward-seeking behaviours in the presence of the aversive cue-indicating that some aspects of the threat memory were preserved ${ }^{70}$. As memories become more distributed or complex, or are assessed with more diverse measures, the effects of interfering with reconsolidation may increasingly look more like updating than substitution of memory ${ }^{51}$. In humans, introducing new information after reactivating an older episodic memory typically leads to integration of this information into the old memory, such that its retrieval now includes some of the new information $^{71}$. Often, retrieval of the older memory content is unimpaired despite the intrusion of new learning-although the stronger the new memory, the more likely impairment in retrieving information from the original memory becomes ${ }^{72}$. Original memories that are older and more narrative (for example, a story as opposed to a list of words) show a stronger influence of new information presented after reactivation on the quality and content of memory for the original event ${ }^{73}$. Distortions and modifications of episodic memory have been documented previously ${ }^{56}$, but an emerging mechanistic understanding of the processes that lead to these changes allows researchers to take advantage of the natural malleability of memory to edit unique memories.

Research on reconsolidation demonstrates that memories can be edited long after a memory is initially learned. Although targeting human reconsolidation appears to modify but not erase memories, this is the closest researchers have come to the kind of memory editing that we see in films. However, it has been two decades since interest in reconsolidation was first reinvigorated ${ }^{40}$, and no clear improvements in clinical treatment have yet been achieved. What these two decades have shown is that reconsolidation is nuanced, particularly in humans. Progress in the field has been complicated by failures to replicate experiments, different results with small procedural changes, and inconsistencies depending on how memory is assessed ${ }^{48,51,74-76}$. In response, researchers have investigated the boundary conditions for targeting reconsolidation, attempting to determine when memory retrieval leads to lability (and when it does not), how the age, strength and complexity of a memory influence its susceptibility to editing and how the interaction of different types of memory expression may affect the success of memory editing ${ }^{51,76-78}$. In addition, without markers of memory lability or synaptic plasticity in humans, it is hard to know whether a memory can be, or has been, successfully edited, or if its expression has instead been temporarily altered by inhibition or new learning. Until some of these issues are addressed, it may be challenging to effectively apply techniques that target reconsolidation to clinical interventions ${ }^{8}$.

\section{Integrating memory editing and clinical therapies}

Many psychiatric disorders are associated with maladaptive memories and/or memory processes. Clinical approaches to treatment often therefore aim to change memory in one form or another (see Box 2). However, only a few clinical strategies focus on targeting memory for a single, circumscribed emotional event. Given that most laboratory editing techniques have assessed unique memories, these strategies might

\section{Box 2 \\ Memory in the clinic}

Most clinical approaches to the treatment of psychiatric disorders deal with memory, especially emotional memory. Psychoanalysis, the traditional form of psychodynamic treatment, assumes that almost everything we learn is permanently stored in the mind as highly preserved, detailed and almost complete records of our past, but that some details may not be accessible all the time ${ }^{96}$. This memory-permanence hypothesis is also known as the 'videorecorder model' 97 . Through hypnosis and other strategies such as dream analysis and free association, Sigmund Freud believed that he would be able to reactivate and access the hidden memories in his patients, and thereby 'work through' the associated conflicts.

Other psychotherapies, such as cognitive behavioural therapies (popularized by Aaron T. Beck ${ }^{98}$ ), similarly acknowledge the importance of emotional events in a person's past and relate them to the psychological distress that the person is experiencing in the present. The goal of these treatments is to identify and correct maladaptive thinking styles that originate from negative experiences that form emotional memories. For example, memories of being rejected or abandoned by key people might lead to the negative cognitive schema of 'I am unlovable'. This schema, in turn, might give rise to automatic thoughts in a specific situation that are associated with emotional distress, defensive behaviours and/or maladaptive habitual actions, thus maintaining the psychological problems that the person experiences. Psychotherapeutic strategies such as these primarily rely on verbal linguistic techniques to discuss and re-evaluate emotional memories.

Contemporary treatments involve exposing patients to emotional memories, either in vivo, using imagery techniques or in a virtual environment. More recent cognitively oriented treatments of trauma include encouraging the development of skills to identify and challenge distorted beliefs that are assumed to maintain the emotional distress associated with the memory. These treatments focus on specific aspects of the memory-the so-called 'stuck points' - that are experienced as especially traumatic, often because of issues related to lack of safety, control, trust, intimacy or power ${ }^{99}$. 


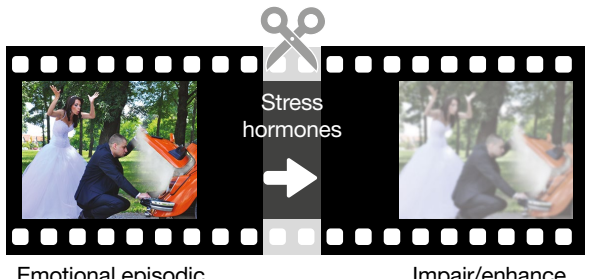

Emotional episodic memory

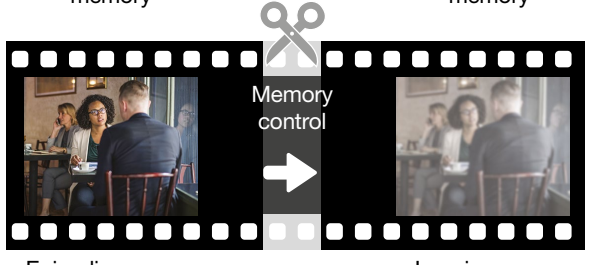

Episodic memory
Impair memory

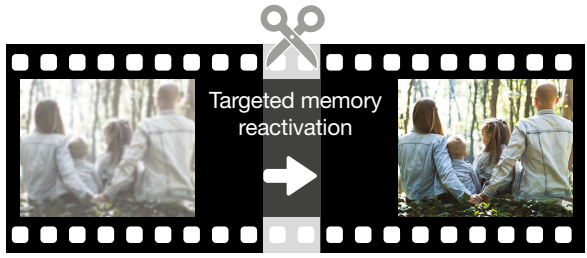

Episodic memory

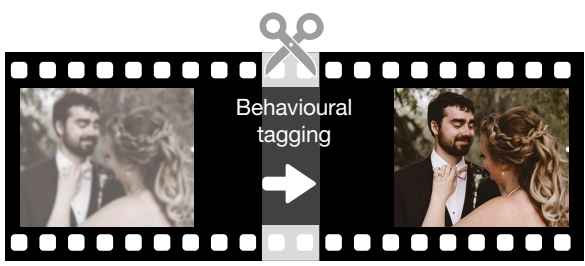

Episodic memory
Enhance memory

b Editing reconsolidation

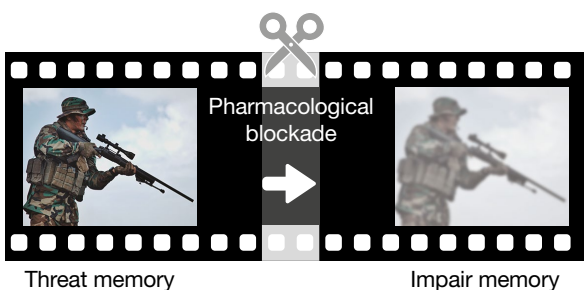

Threat memory

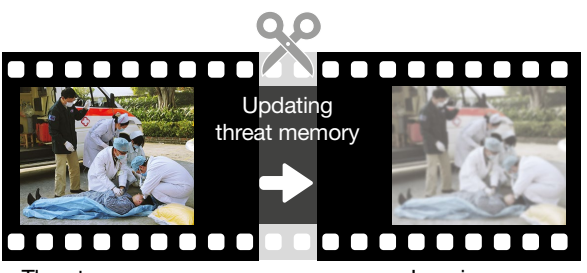

Threat memory

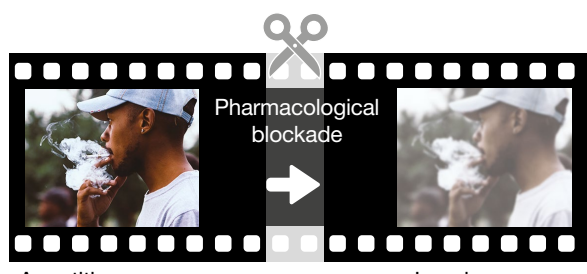

Appetitive memory

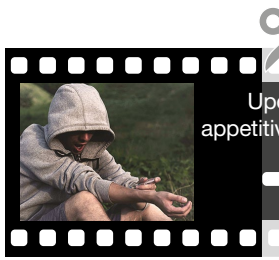

Appetitive memory
0.0

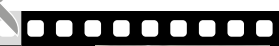
dating etitive memory $\gamma$ 10000
Fig. 2 Memory-editing techniques. a, Four primary techniques have been investigated to edit the consolidation of memories in humans. Research using these techniques has primarily focused on editing episodic memory. Manipulations of stress hormones both impair emotional memories and enhance neutral memories; targeted memory reactivation and behavioural tagging enhance memory; and memory control impairs memory. b, Pharmacological and behavioural interventions have been

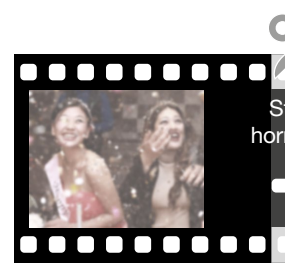

Emotional episodic memory

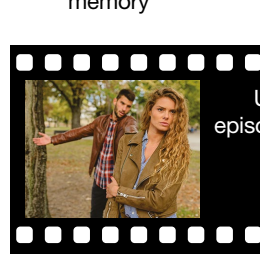

Episodic memory
00

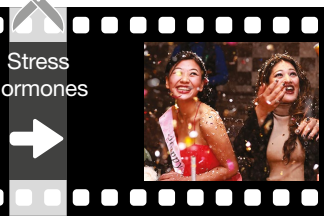

Impair/enhance memory

0.9

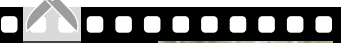
Updating isodic memory

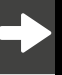
0 00000000 Integrate/impair memory

used to edit the reconsolidation of memories. Pharmacological blockade and updating the reconsolidation of threat and appetitive memories diminish learned defensive responses and appetitive behaviours, respectively. Similar to consolidation, stress hormones that target reconsolidation can both enhance neutral memories and impair emotional memories. Updating episodic memory reconsolidation can both modify memory content and impair memory.

be the best starting point for introducing memory-editing techniques to the clinic.

As discussed above, this approach has already been attempted with exposure therapy. The aim of all exposure-therapy techniques is to reduce the emotional distress associated with a trauma memory by repeatedly experiencing and thereby emotionally processing the traumatic memory through exposures. Much like extinction training, repeated exposures are assumed to promote new safety learning that inhibits the original trauma memory, rather than editing it $^{79}$.

For example, in prolonged exposure therapy, the patient is repeatedly confronted with the trauma memory, usually daily or several times per week, for an extended period of time (often 30 minutes or longer) ${ }^{80}$. Prolonged exposure is based on the notion that the memory is part of a pathological fear structure that maintains the emotional distress associated with the trauma. To reduce pathological fear, the emotional processing theory ${ }^{81}$ suggests that the fear structure must first be activated, and corrective information that is incompatible with the pathological elements of the memory structure must then be incorporated. Therefore, cognitive changes are considered necessary for prolonged exposure therapy to be effective.

Because of the overlap between laboratory extinction paradigms and exposure therapies, integrating editing techniques into this type of clinical therapy is relatively straightforward. So far, this approach has shown little to modest success in preclinical studies ${ }^{82}$. Optimizing the integration of memory editing into exposure therapy will require the identification of methods to overcome the boundaries of reconsolidation $^{8}$, as well as an understanding of how targeting reconsolidation of one memory type (such as defensive responses) alters other means of memory expression (such as negative subjective feelings and episodic memory).

Imagery rescripting is another therapeutic strategy that targets unique emotional memories ${ }^{83}$. In imagery rescripting, patients are asked to vividly imagine a traumatic event, but then to change the outcome or details of the event to make it non-traumatic and desirable. For example, a woman who had been raped might imagine that she develops supernatural powers and throws the rapist out of the window; or she might imagine that a police officer enters the room to arrest the rapist before the rape occurs. This rescripted scenario is not usually based on reality, or even realistic. Rather, it is an alternative and desirable story that shares many of the features of the original trauma memory. The mechanism of imagery rescripting is not well understood $^{83}$. However, given that it involves unique memory alterations, this approach could potentially be enhanced by integrating memory-editing techniques. 
The status and future of memory editing in the clinic The idea that human memories can be selectively targeted for editing is no longer just science fiction. However, in this case, truth (or research) is stranger, more complicated and more nuanced than fiction. Unlike in films, current memory-editing techniques appear to modify aspects of memories, rather than erase them. These modifications may be subtle at times, but could still be of clinical consequence. Relatively little is known as yet about how any of the techniques outlined in this Review might most effectively be applied to clinical treatments. Innovative approaches to identifying synaptic plasticity and memory lability in humans, or translating interventions that are now limited to animal models to humans, will strengthen our ability to take advantage of memory editing. Equally important will be a more detailed understanding of how memory influences psychopathology ${ }^{84}$. Without a clear picture of the effect of memory on psychiatric disorders, we will not know when to use innovations in editing to improve treatment outcomes.

Although current efforts to incorporate memory editing into clinical interventions have yielded inconsistent benefits, there is reason for optimism. There are a number of memory-editing techniques that are effective in laboratory paradigms (Fig. 2), and many approaches have yet to be investigated in the clinic. Advances in human brain science will provide new insights into exactly how editing changes memory and what can be done to increase its effectiveness. The science of memory editing is rapidly progressing and new insights are emerging regularly. Given this, it is probable that robust, easily implemented techniques to edit unique human memories in the clinic and beyond will be developed. However, just like in the movies, we may find that if we succeed in easily editing human memories, there could be unexpected consequences for how we think about memory and its role in defining who we are.

Received: 22 February 2019; Accepted: 17 June 2019;

Published online 31 July 2019.

1. Kandel, E. R., Dudai, Y. \& Mayford, M. R. The molecular and systems biology of memory. Cell 157, 163-186 (2014).

2. Wood, N. E. et al. Pharmacological blockade of memory reconsolidation in posttraumatic stress disorder: three negative psychophysiological studies. Psychiatry Res. 225, 31-39 (2015).

3. Xue, Y. X. et al. A memory retrieval-extinction procedure to prevent drug craving and relapse. Science 336, 241-245 (2012)

4. Roediger, H. L., III \& Butler, A. C. The critical role of retrieval practice in long-term retention. Trends Cogn. Sci. 15, 20-27 (2011).

5. Kim, W. B. \& Cho, J. H. Encoding of discriminative fear memory by inputspecific LTP in the amygdala. Neuron 95, 1129-1146 (2017).

6. Trouche, S. et al. Recoding a cocaine-place memory engram to a neutral engram in the hippocampus. Nat. Neurosci. 19, 564-567 (2016).

7. Lane, R. D., Ryan, L., Nadel, L. \& Greenberg, L. Memory reconsolidation, emotional arousal, and the process of change in psychotherapy: new insights from brain science. Behav. Brain Sci. 38, e1 (2015).

8. Monfils, M. H. \& Holmes, E. A. Memory boundaries: opening a window inspired by reconsolidation to treat anxiety, trauma-related, and addiction disorders. Lancet Psychiatry 5, 1032-1042 (2018).

This is a recent review of research that attempts to translate manipulations of reconsolidation to the clinic.

9. Dudai, Y., Karni, A. \& Born, J. The consolidation and transformation of memory. Neuron 88, 20-32 (2015).

10. McGaugh, J. L. Memory - a century of consolidation. Science 287, 248-251 (2000). This paper reviews the neurobiology of the role of stress hormones in modulating consolidation.

11. LaBar, K. S. \& Phelps, E. A. Arousal-mediated memory consolidation: role of the medial temporal lobe in humans. Psychol. Sci. 9, 490-493 (1998).

12. Cahill, L., Gorski, L. \& Le, K. Enhanced human memory consolidation with post-learning stress: interaction with the degree of arousal at encoding. Learn. Mem. 10, 270-274 (2003)

13. Cahill, L., Prins, B., Weber, M. \& McGaugh, J. L. $\beta$-adrenergic activation and memory for emotional events. Nature 371, 702-704 (1994).

14. Mayou, R. A., Ehlers, A. \& Hobbs, M. Psychological debriefing for road traffic accident victims. Three-year follow-up of a randomised controlled trial. Br. J. Psychiatry 176, 589-593 (2000).

15. Sijbrandij, M., Olff, M., Reitsma, J. B., Carlier, I. V. \& Gersons, B. P. Emotional or educational debriefing after psychological trauma: randomised controlled trial. Br. J. Psychiatry 189, 150-155 (2006)

16. Hoge, E. A. et al. Effect of acute posttrauma propranolol on PTSD outcome and physiological responses during script-driven imagery. CNS Neurosci. Ther. 18, 21-27 (2012)
17. Soravia, L. M. et al. Glucocorticoids enhance in vivo exposure-based therapy of spider phobia. Depress. Anxiety 31, 429-435 (2014).

18. Yehuda, R. et al. Cortisol augmentation of a psychological treatment for warfighters with posttraumatic stress disorder: randomized trial showing improved treatment retention and outcome. Psychoneuroendocrinology 51, 589-597 (2015)

19. Bouton, M. E. Context and behavioral processes in extinction. Learn. Mem. 11, 485-494 (2004).

20. Dunsmoor, J. E., Niv, Y., Daw, N. \& Phelps, E. A. Rethinking extinction. Neuron 88 47-63 (2015).

21. Vervliet, B., Craske, M. G. \& Hermans, D. Fear extinction and relapse: state of the art. Annu. Rev. Clin. Psychol. 9, 215-248 (2013).

22. Surís, A., North, C., Adinoff, B., Powell, C. M. \& Greene, R. Effects of exogenous glucocorticoid on combat-related PTSD symptoms. Ann. Clin. Psychiatry 22, 274-279 (2010).

23. Rasch, B. \& Born, J. Maintaining memories by reactivation. Curr. Opin. Neurobiol. 17, 698-703 (2007)

24. Tambini, A., Berners-Lee, A. \& Davachi, L. Brief targeted memory reactivation during the awake state enhances memory stability and benefits the weakest memories. Sci. Rep. 7, 15325 (2017).

25. Alm, K. H., Ngo, C. T. \& Olson, I. R. Hippocampal signatures of awake targeted memory reactivation. Brain Struct. Funct. 224, 713-726 (2019).

26. Lehmann, M., Schreiner, T., Seifritz, E. \& Rasch, B. Emotional arousal modulates oscillatory correlates of targeted memory reactivation during NREM, but not REM sleep. Sci. Rep. 6, 39229 (2016).

27. Hauner, K. K., Howard, J. D., Zelano, C. \& Gottfried, J. A. Stimulus-specific enhancement of fear extinction during slow-wave sleep. Nat. Neurosci. 16, 1553-1555 (2013).

28. $\mathrm{He}, \mathrm{J}$. et al. Effect of conditioned stimulus exposure during slow wave sleep on fear memory extinction in humans. Sleep 38, 423-431 (2015).

29. Simon, K. C. N. S., Gómez, R. L. \& Nadel, L. Losing memories during sleep after targeted memory reactivation. Neurobiol. Learn. Mem. 151, 10-17 (2018).

30. Hu, X., Bergström, Z. M., Gagnepain, P. \& Anderson, M. C. Suppressing unwanted memories reduces their unintended influences. Curr. Dir. Psychol. Sci. 26, 197-206 (2017).

31. Hulbert, J. C. \& Anderson, M. C. What doesn't kill you makes you stronger: psychological trauma and its relationship to enhanced memory control. J. Exp. Psychol. Gen. 147, 1931-1949 (2018).

32. Schmitz, T. W., Correia, M. M., Ferreira, C. S., Prescot, A. P. \& Anderson, M. C. Hippocampal GABA enables inhibitory control over unwanted thoughts. Nat. Commun. 8, 1311 (2017)

33. Gagnepain, P., Henson, R. N. \& Anderson, M. C. Suppressing unwanted memories reduces their unconscious influence via targeted cortical inhibition. Proc. Natl Acad. Sci. USA 111, E1310-E1319 (2014).

34. Wang, S. H., Redondo, R. L. \& Morris, R. G. Relevance of synaptic tagging and capture to the persistence of long-term potentiation and everyday spatial memory. Proc. Natl Acad. Sci. USA 107, 19537-19542 (2010).

35. Dunsmoor, J. E., Murty, V. P., Davachi, L. \& Phelps, E. A. Emotional learning selectively and retroactively strengthens memories for related events. Nature 520, 345-348 (2015).

36. Patil, A., Murty, V. P., Dunsmoor, J. E., Phelps, E. A. \& Davachi, L. Reward retroactively enhances memory consolidation for related items. Learn. Mem. 24, 65-69 (2017).

37. Nomoto, M. et al. Cellular tagging as a neural network mechanism for behavioural tagging. Nat. Commun. 7, 12319 (2016).

38. Dudai, Y. The restless engram: consolidations never end. Annu. Rev. Neurosci. 35, 227-247 (2012).

39. Misanin, J. R., Miller, R. R. \& Lewis, D. J. Retrograde amnesia produced by electroconvulsive shock after reactivation of a consolidated memory trace. Science 160, 554-555 (1968).

40. Nader, K., Schafe, G. E. \& Le Doux, J. E. Fear memories require protein synthesis in the amygdala for reconsolidation after retrieval. Nature 406, 722-726 (2000).

This study launched the reinvigoration of research into the neurobiology of reconsolidation.

41. Dębiec, J. \& LeDoux, J. E. Disruption of reconsolidation but not consolidation of auditory fear conditioning by noradrenergic blockade in the amygdala. Neuroscience 129, 267-272 (2004)

42. Kroes, M. C. W., Schiller, D., LeDoux, J. E. \& Phelps, E. A. Translational approaches targeting reconsolidation. Curr. Top. Behav. Neurosci. 28, 197-230 (2016).

43. Kindt, M., Soeter, M. \& Vervliet, B. Beyond extinction: erasing human fear responses and preventing the return of fear. Nat. Neurosci. 12, 256-258 (2009).

44. Soeter, M. \& Kindt, M. An abrupt transformation of phobic behavior after a post-retrieval amnesic agent. Biol. Psychiatry 78, 880-886 (2015).

45. Pitman, R. K. Harnessing reconsolidation to treat mental disorders. Biol. Psychiatry 78, 819-820 (2015).

46. Brunet, A. et al. Reduction of PTSD symptoms with pre-reactivation propranolo therapy: a randomized controlled trial. Am. J. Psychiatry 175, 427-433 (2018).

47. Lee, J. L. C., Di Ciano, P., Thomas, K. L. \& Everitt, B. J. Disrupting reconsolidation of drug memories reduces cocaine-seeking behavior. Neuron 47, 795-801 (2005)

48. Lee, J. L. \& Everitt, B. J. Reactivation-dependent amnesia in Pavlovian approach and instrumental transfer. Learn. Mem. 15, 597-602 (2008).

49. Fricks-Gleason, A. N. \& Marshall, J. F. Post-retrieval $\beta$-adrenergic receptor blockade: effects on extinction and reconsolidation of cocaine-cue memories. Learn. Mem. 15, 643-648 (2008). 
50. Saladin, M. E. et al. A double blind, placebo-controlled study of the effects of post-retrieval propranolol on reconsolidation of memory for craving and cue reactivity in cocaine dependent humans. Psychopharmacology 226, 721-737 (2013).

51. Schiller, D. \& Phelps, E. A. Does reconsolidation occur in humans? Front. Behav. Neurosci. 5, 24 (2011).

52. Alberini, C. M. \& Ledoux, J. E. Memory reconsolidation. Curr. Biol. 23, R746-R750 (2013)

53. Bos, M. G. N., Schuijer, J., Lodestijn, F., Beckers, T. \& Kindt, M. Stress enhances reconsolidation of declarative memory. Psychoneuroendocrinology 46, 102-113 (2014).

54. Kroes, M. C. et al. An electroconvulsive therapy procedure impairs reconsolidation of episodic memories in humans. Nat. Neurosci. 17, 204-206 (2014).

55. Squire, L. R. ECT and memory loss. Am. J. Psychiatry 134, 997-1001 (1977).

56. Schacter, D. L., Guerin, S. A. \& St Jacques, P. L. Memory distortion: an adaptive perspective. Trends Cogn. Sci. 15, 467-474 (2011).

57. Monfils, M. H., Cowansage, K. K., Klann, E. \& LeDoux, J. E. Extinctionreconsolidation boundaries: key to persistent attenuation of fear memories. Science 324, 951-955 (2009).

This study demonstrated that a behavioural intervention can target threat memory reconsolidation and highlighted that not every retrieval results in evidence of synaptic plasticity.

58. Fernández, R. S., Boccia, M. M. \& Pedreira, M. E. The fate of memory: reconsolidation and the case of prediction error. Neurosci. Biobehav. Rev. 68 423-441 (2016).

59. Gershman, S. J., Monfils, M. H., Norman, K. A. \& Niv, Y. The computational nature of memory modification. eLife 6, e23763 (2017)

This study is a theoretical account of when reactivation leads to memory lability and when it does not.

60. Schiller, D. et al. Preventing the return of fear in humans using reconsolidation update mechanisms. Nature 463, 49-53 (2010).

61. Schiller, D., Kanen, J. W., LeDoux, J. E., Monfils, M. H. \& Phelps, E. A. Extinction during reconsolidation of threat memory diminishes prefrontal cortex involvement. Proc. Natl Acad. Sci. USA 110, 20040-20045 (2013).

62. Shiban, Y., Brütting, J., Pauli, P. \& Mühlberger, A. Fear reactivation prior to exposure therapy: does it facilitate the effects of VR exposure in a randomized clinical sample? J. Behav. Ther. Exp. Psychiatry 46, 133-140 (2015).

63. Telch, M. J., York, J., Lancaster, C. L. \& Monfils, M. H. Use of a brief fear memory reactivation procedure for enhancing exposure therapy. Clin. Psychol. Sci. 5, 367-378 (2017).

64. Kredlow, M. A. \& Otto, M. W. Interference with the reconsolidation of traumarelated memories in adults. Depress. Anxiety 32, 32-37 (2015).

65. Gray, R., Budden-Potts, D. \& Bourke, F. Reconsolidation of traumatic memories for PTSD: a randomized controlled trial of 74 veterans. Psychother. Res. 29 621-639 (2019).

66. James, E. L. et al. Computer game play reduces intrusive memories of experimental trauma via reconsolidation-update mechanisms. Psychol. Sci. 26 1201-1215 (2015)

67. Cofresí, R. U. et al. Postretrieval extinction attenuates alcohol cue reactivity in rats. Alcohol. Clin. Exp. Res. 41, 608-617 (2017)

68. Sartor, G. C. \& Aston-Jones, G. Post-retrieval extinction attenuates cocaine memories. Neuropsychopharmacology 39, 1059-1065 (2014).

69. Germeroth, L. J. et al. Effect of a brief memory updating intervention on smoking behavior: a randomized clinical trial. JAMA Psychiatry 74, 214-223 (2017).

70. Shumake, J. \& Monfils, M. H. Assessing fear following retrieval + extinction through suppression of baseline reward seeking vs. freezing. Front. Behav. Neurosci. 9, 355 (2015).

71. Hupbach, A., Gomez, R., Hardt, O. \& Nadel, L. Reconsolidation of episodic memories: a subtle reminder triggers integration of new information. Learn. Mem. 14, 47-53 (2007).

72. Wichert, S., Wolf, O. T. \& Schwabe, L. Updating of episodic memories depends on the strength of new learning after memory reactivation. Behav. Neurosci. 127, 331-338 (2013).

73. Scully, I. D., Napper, L. E. \& Hupbach, A. Does reactivation trigger episodic memory change? A meta-analysis. Neurobiol. Learn. Mem. 142, 99-107 (2017). This is a meta-analysis and review on the updating of episodic memory.

74. Schroyens, N., Beckers, T. \& Kindt, M. In search for boundary conditions of reconsolidation: a failure of fear memory interference. Front. Behav. Neurosci. 11,65 (2017)

75. Bos, M. G., Beckers, T. \& Kindt, M. Noradrenergic blockade of memory reconsolidation: a failure to reduce conditioned fear responding. Front. Behav. Neurosci. 8, 412 (2014)

76. Auber, A., Tedesco, V., Jones, C. E., Monfils, M. H. \& Chiamulera, C. Post-retrieval extinction as reconsolidation interference: methodological issues or boundary conditions? Psychopharmacology 226, 631-647 (2013)

77. Dunbar, A. B. \& Taylor, J. R. Reconsolidation and psychopathology: moving towards reconsolidation-based treatments. Neurobiol. Learn. Mem. 142, 162-171 (2017).

78. Kroes, M. C. W., Dunsmoor, J. E., Lin, Q., Evans, M. \& Phelps, E. A. A reminder before extinction strengthens episodic memory via reconsolidation but fails to disrupt generalized threat responses. Sci. Rep. 7, 10858 (2017).
79. Craske, M. G., Treanor, M., Conway, C. C., Zbozinek, T. \& Vervliet, B. Maximizing exposure therapy: an inhibitory learning approach. Behav. Res. Ther. 58, 10-23 (2014).

80. Foa, E. B., Hembree, E. \& Rothbaum, B. Prolonged Exposure Therapy for PTSD: Emotional Processing of Traumatic Experiences: Therapist Guide (Oxford Univ. Press, 2007).

81. Foa, E. B. \& Kozak, M. J. Emotional processing of fear: exposure to corrective information. Psychol. Bull. 99, 20-35 (1986).

82. Walsh, K. H., Das, R. K., Saladin, M. E. \& Kamboj, S. K. Modulation of naturalistic maladaptive memories using behavioural and pharmacological reconsolidation-interfering strategies: a systematic review and meta-analysis of clinical and 'sub-clinical' studies. Psychopharmacology 235, 2507-2527 (2018).

83. Arntz, A. Imagery rescripting as a therapeutic techniques: review of clinical trials, basic studies, and research agenda. J. Exp. Psychopathol. 3, 189-208 (2012).

84. Engelhard, I. M., McNally, R. J. \& van Schie, K. Retrieving and modifying traumatic memories: recent research relevant to three controversies. Curr. Dir. Psychol. Sci. 28, 91-96 (2019). This is a discussion of issues that are relevant to the clinical translation of memory editing.

85. Brewer, D., Doughtie, E. B. \& Lubin, B. Induction of mood and mood shift. J. Clin. Psychol. 36, 215-226 (1980)

86. Josephson, B. R. Mood regulation and memory: repairing sad moods with happy memories. Cogn. Emot. 10, 437-444 (1996).

87. Speer, M. E. \& Delgado, M. R. Reminiscing about positive memories buffers acute stress responses. Nat. Hum. Behav. 1, 0093 (2017)

88. Lempert, K. M., Speer, M. E., Delgado, M. R. \& Phelps, E. A. Positive autobiographical memory retrieval reduces temporal discounting. Soc. Cogn. Affect. Neurosci. 12, 1584-1593 (2017).

89. Hofmann, W., De Houwer, J., Perugini, M., Baeyens, F. \& Crombez, G. Evaluative conditioning in humans: a meta-analysis. Psychol. Bull. 136 390-421 (2010)

90. Baeyens, F., Díaz, E. \& Ruiz, G. Resistance to extinction of human evaluative conditioning using a between-subjects design. Cogn. Emot. 19, 245-268 (2005).

91. Benedict, T., Richter, J. \& Gast, A. The influence of misinformation manipulations on evaluative conditioning. Acta Psychol. 194, 28-36 (2019).

92. Raio, C. M., Orederu, T. A., Palazzolo, L., Shurick, A. A. \& Phelps, E. A. Cognitive emotion regulation fails the stress test. Proc. Natl Acad. Sci. USA 110, 15139-15144 (2013)

93. Kross, E., Davidson, M., Weber, J. \& Ochsner, K. Coping with emotions past: the neural bases of regulating affect associated with negative autobiographical memories. Biol. Psychiatry 65, 361-366 (2009).

94. Doré, B. P. et al. Negative autobiographical memory in depression reflects elevated amygdala-hippocampal reactivity and hippocampally associated emotion regulation. Biol. Psychiatry Cogn. Neurosci. Neuroimaging 3, 358-366 (2018).

95. Hirst, W. et al. Long-term memory for the terrorist attack of September 11: flashbulb memories, event memories, and the factors that influence their retention. J. Exp. Psychol. Gen. 138, 161-176 (2009).

96. Freud, S. Freud's Psycho-Analytic Procedure. The Standard Edition of the Complete Psychological Works of Sigmund Freud Vol. 7 249-254 (International Univ. Press, 1904)

97. Loftus, E. F. \& Loftus, G. R. On the permanence of stored information in the human brain. Am. Psychol. 35, 409-420 (1980).

98. Beck, A. T. Cognitive Therapy and the Emotional Disorders (New American Library and Meridian, 1979).

99. Ehlers, A. \& Clark, D. M. A cognitive model of posttraumatic stress disorder. Behav. Res. Ther. 38, 319-345 (2000).

Acknowledgements We acknowledge support from the James S. McDonnell Foundation and assistance from S. Aamodt and E. Zhukovsky in the preparation of this manuscript.

Reviewer information Nature thanks Richard Lane and the other, anonymous, reviewer(s) for their contribution to the peer review of this work.

Author contributions E.A.P. wrote the majority of the first draft and S.G.H. provided contributions on clinical approaches. Both E.A.P. and S.G.H. edited the manuscript. E.A.P. wrote Box 1 and S.G.H. wrote Box 2

Competing interests The authors declare no competing interests.

\section{Additional information}

Reprints and permissions information is available at http://www.nature.com/ reprints.

Correspondence and requests for materials should be addressed to E.A.P.

Publisher's note: Springer Nature remains neutral with regard to jurisdictional claims in published maps and institutional affiliations.

(C) Springer Nature Limited 2019 